\title{
Assessment of RANS Turbulence Models for Straight Cooling Ducts: Secondary Flow and Strong Property Variation Effects
}

\author{
Thomas Kaller, Alexander Doehring, Stefan Hickel, Steffen J. Schmidt, \\ and Nikolaus A. Adams
}

\begin{abstract}
We present well-resolved RANS simulations of two generic asymmetrically heated cooling channel configurations, a high aspect ratio cooling duct operated with liquid water at $R e_{b}=110 \times 10^{3}$ and a cryogenic transcritical channel operated with methane at $R e_{b}=16 \times 10^{3}$. The former setup serves to investigate the interaction of turbulence-induced secondary flow and heat transfer, and the latter to investigate the influence of strong non-linear thermodynamic property variations in the vicinity of the critical point on the flow field and heat transfer. To assess the accuracy of the RANS simulations for both setups, well-resolved implicit LES simulations using the adaptive local deconvolution method as subgrid-scale turbulence model serve as comparison databases. The investigation focuses on the prediction capabilities of RANS turbulence models for the flow as well as the temperature field and turbulent heat transfer with a special focus on the turbulent heat flux closure influence.
\end{abstract}

\section{Introduction}

Understanding cooling duct flows is essential for efficient structural cooling in many technical applications. Examples range from ventilation systems, electrical component cooling to launcher propulsion systems. The latter use the cryogenic propellant as coolant at a supercritical state.

T. Kaller $(\bowtie) \cdot$ A. Doehring $(\bowtie) \cdot$ S. J. Schmidt $\cdot$ N. A. Adams

Chair of Aerodynamics and Fluid Mechanics, Department of Mechanical Engineering,

Technical University of Munich, Boltzmannstr. 15, D-85748 Garching bei München, Germany

e-mail: thomas.kaller@tum.de

A. Doehring

e-mail: alex.doehring@tum.de

S. Hickel

Faculty of Aerospace Engineering, Technische Universiteit Delft, Kluyverweg 1,

2629 HT Delft, The Netherlands 
The turbulent flow and heat transfer within a cooling duct is highly affected by the presence of secondary flows and strong non-linear thermodynamic property variations in the vicinity of the pseudo-boiling line (PBL) [5]. Secondary flows enhance the mixing of hot and cold fluid and increase thus the overall cooling efficiency. Within the current study we focus on the relatively weak turbulence-induced secondary flow. Strong non-linear property variations are induced by intermolecular repulsive forces and significantly affect the heat transfer and shear forces. As a consequence, effects like the heat transfer enhancement as well as the onset of heat transfer deterioration in transcritical flows are difficult to correctly predict.

Three major turbulence simulation classes exist: direct numerical simulations (DNS), large-eddy simulations (LES) and Reynolds-averaged Navier-Stokes simulations (RANS). In DNS all spatial and temporal scales are fully resolved. In LES, large turbulent structures are resolved, whereas small scales or subgrid-scales (SGS) are modelled. Using RANS, the Navier-Stokes equations (NSE) are solved approximately for the averaged state and all scales are modelled. To close the equation system approximations for Reynolds stresses $\overline{u_{i}^{\prime} u_{j}^{\prime}}$ and turbulent heat fluxes $\overline{u_{i}^{\prime} h^{\prime}}$ have to be derived. Reynolds stress models (RSM) introduce partial differential equations (PDE) for the individual turbulent stress components offering the advantage over less complex models, like the $k-\varepsilon$ or SST models, to account for turbulence anisotropy. To approximate the unknown turbulent heat fluxes the most prevalent method is using a gradient transport approach with a constant $P r_{t}$. To account for the anisotropy of $\overline{u_{i}^{\prime} h^{\prime}}$ additional PDEs can be introduced for the individual components or a less expensive algebraic approximation based on the Reynolds stress tensor utilised.

Relevant DNS studies of turbulence-induced secondary flow in square ducts include [11, 25], and in high aspect ratio ducts [31], the $A R$ ranging from $1-7$. The interaction of heating and turbulence-induced secondary flow has been analysed by [29] for square ducts and by [6] for rectangular ducts at small aspect ratios, both performed LES. DNS of a transcritical channel flow has been performed by Ma et al. [24] using an entropy-stable double-flux model in order to avoid spurious pressure oscillations. They have observed a logarithmic scaling of the second-order structure function and a $k^{-1}$ scaling of the streamwise energy spectra, which supports the attached-eddy hypothesis in transcritical flows. A heated transcritical turbulent boundary layer over a flat plate has been investigated by Kawai [20] with DNS. His study shows large density fluctuations, which exceed Morkovin's hypothesis and lead to a non-negligible turbulent mass flux. RANS studies for cooling duct flows under realistic rocket engine conditions have been presented by [26, 27].

In the first part of the present study an asymmetrically heated high aspect ratio cooling duct (HARCD) at $R e_{b}=110 \cdot 10^{3}$ and a moderate heating of $T_{W}-T_{b}=$ $40 \mathrm{~K}$ is investigated using the BSL RSM and various turbulent heat flux closure models with ANSYS CFX. This setup has been studied experimentally, [28], and using a LES, [16-19] serving as comparison database. In the second part a cryogenic transcritical channel at $R e_{b}=16 \cdot 10^{3}$ is investigated with the BSL RSM and various turbulent heat flux closure models with ANSYS FLUENT. The bulk pressure surpasses the critical value and the wall temperatures enclose the pseudo-boiling temperature. This setup has been studied in $[8,9]$ serving as comparison database. 
The overall target is to assess the prediction capability of industrial RANS tools for cooling duct flows with a focus on the influence of the turbulent heat flux closure.

\section{High Aspect Ratio Cooling Duct}

This section focuses on the asymmetrically heated HARCD. Results for the RANS BSL RSM in combination with different heat flux closure models are compared to a LES to assess the prediction capability of secondary flow and turbulent heat transfer.

\subsection{Equation System and Numerical Model}

For the RANS simulations the compressible Navier-Stokes equations (NSE) with the total energy equation are used as implemented in ANSYS CFX, see [1, 2]. The fluid properties are evaluated based on the IAPWS IF97 formulation. To close the equation system approximations for $\rho \overline{u_{i}^{\prime} u_{j}^{\prime}}$ and $\rho \overline{u_{i}^{\prime} h^{\prime}}$ are required. Reference [17] showed, that the $\omega$-based BSL RSM gives the overall best results for the HARCD setup. Additional PDEs are solved for each component of $\rho \overline{u_{i}^{\prime} u_{j}^{\prime}}$ and the specific dissipation $\omega$. At the walls the so-called automatic wall treatment functionality is employed.

For modelling $\rho \overline{u_{i}^{\prime} h^{\prime}}$ we utilise the state of the art gradient approach with a constant $P r_{t}$, two algebraic and a second moment closure model. For the gradient approach the turbulent heat fluxes are proportional to the enthalpy gradients and the isotropic turbulent diffusivity $\alpha_{t}$ with $\alpha_{t}=v_{t} / P r_{t}$. The algebraic Daly-Harlow and the improved Younis models employ an anisotropic $\alpha_{t}$-tensor as a function of the Reynolds stress tensor, see [7, 32]. For the second moment closure model an additional PDE is solved for each component of $\rho \overline{u_{i}^{\prime} h^{\prime}}$. The latter is a beta feature within ANSYS CFX (CADFEM GmbH, personal communication, 2018).

For the LES database of [19] the incompressible NSE with the Boussinesq approximation are applied. The transport properties are evaluated using the IAPWS correlations. For time discretisation a third-order Runge-Kutta scheme with $C F L=1$ is utilised and for spatial discretisation a second-order finite-volume method. As an implicit LES is performed, the size of the subgrid scales (SGS) is determined by the chosen grid resolution. As SGS model the adaptive local deconvolution method (ALDM) is used, see [13].

\subsection{Simulation Setup}

The setup consists of two domains simulated independently, see Fig. 1. The adiabatic periodic section is $50 \times 25.8 \times 6 \mathrm{~mm}^{3}$ and the heated section is $600 \times 25.8 \times 6 \mathrm{~mm}^{3}$. 


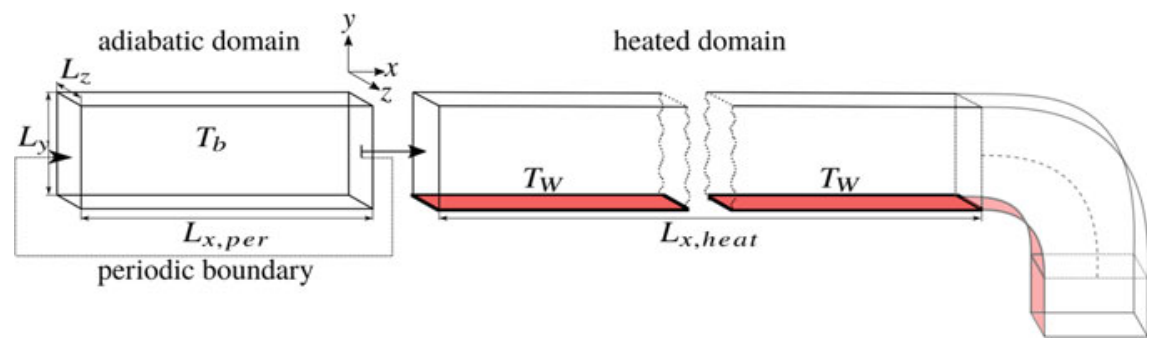

Fig. 1 Simulation setup with the focus of the current investigation on the non-faded part

The straight part is followed by a curved section, which is not part of the current study and analysed in [17]. The grid resolution for the well-resolved RANS has been determined with an extensive grid sensitivity study based on the periodic section and satisfies $y^{+} \approx 1$ for the adiabatic and heated walls. In total 34/512 $\times 115 \times 64$ nodes are used for the periodic, respectively the heated domain.

The periodic duct serves to generate a fully developed turbulent HARCD inflow profile. The simulation is performed with liquid water treated as incompressible with fixed fluid properties at $T_{b}=333.15 \mathrm{~K}$. All walls are defined as smooth adiabatic walls. In streamwise direction a periodic boundary condition is set with a constant mass flow of $\dot{m}=0.8193 \mathrm{~kg} / \mathrm{s}$ corresponding to $u_{b}=5.3833 \mathrm{~m} / \mathrm{s}$ and $R e_{b}=110$. $10^{3}$. Convergence is accelerated by using physical and local time stepping methods and lowering the pressure update multiplier, and is reached when a RMS target value of $1 \cdot 10^{-6}$ is surpassed for the momentum and continuity equation residuals.

For the heated domain simulations the compressible NSE are used. All walls are treated as smooth walls with the automatic wall treatment option applied. The lower wall is an isothermal wall with a fixed $T_{W}=373.15 \mathrm{~K}$ and the remaining are adiabatic walls. At the inlet velocity and turbulence fields from the periodic domain are prescribed, and at the outlet an average pressure of $p_{\text {out }}=101325 \mathrm{~Pa}$ is set. Convergence is accelerated by using physical and local time stepping methods, and is reached when a RMS target value of $1 \cdot 10^{-6}$ is surpassed for the momentum, continuity and total energy equation residuals.

\subsection{Flow and Temperature Field}

In the following, the RANS results of the BSL RSM turbulence model in combination with different turbulent heat flux closures are compared to the LES of [19].

Figure 2 shows the cross-sectional flow and temperature field and Fig. 3 depicts the flow and temperature profiles along the duct midplane at $x=300 \mathrm{~mm}$ after the beginning of the heated straight HARCD. As the choice of turbulent heat flux closure has a negligible effect on the velocity field all RANS results coincide in Fig. $3 \mathrm{a} / \mathrm{b}$. We observe for the streamwise velocity of the adiabatic duct results, that the LES 


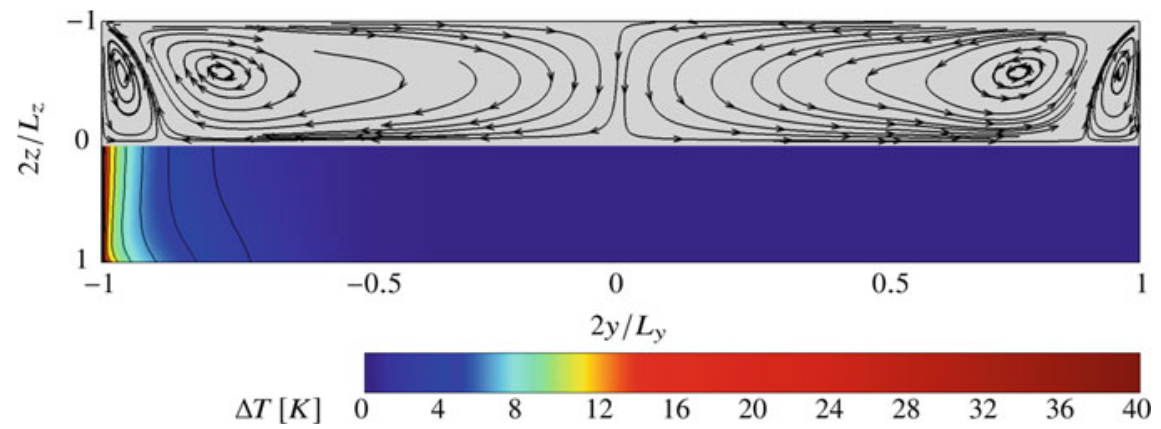

Fig. 2 Secondary flow and temperature field at $x=300 \mathrm{~mm}$ for the BSL RSM with $P r_{t}=0.9$. Isolines are drawn from $2 \mathrm{~K}$ to $40 \mathrm{~K}$ in steps of $2 \mathrm{~K}$
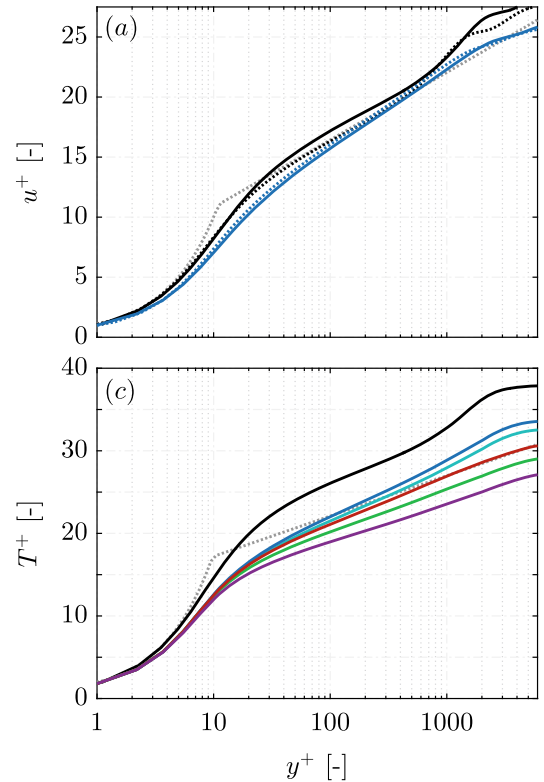
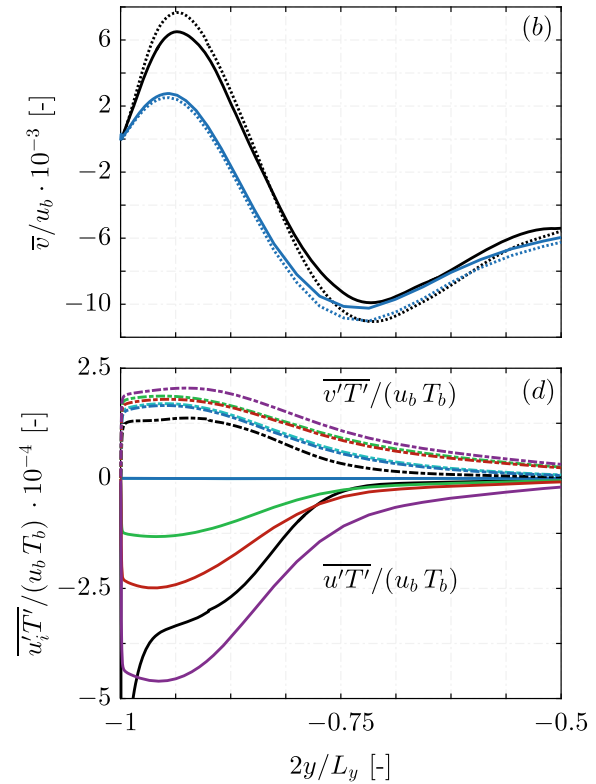

Fig. 3 Streamwise (a) and secondary flow velocity (b), temperature (c) and turbulent heat flux distribution (d) along $2 z / L_{z}=0$ at $x=300 \mathrm{~mm}$ for the LES (- - and the BSL RSM with $P r_{t}=0.85(-), P r_{t}=0.9$ ( $\longrightarrow$ ), Daly-Harlow model $(\square)$, Younis model ( $\longrightarrow$ ) and PDE model ( $\longrightarrow$ ). In $\mathbf{a} / \mathbf{b}$ the adiabatic results are plotted as dotted lines. In $\mathbf{a} / \mathbf{c}$ the analytical law of the wall and the empirical function of Kader are plotted as ( .............

follows closely the analytical law of the wall, $u^{+}=1 / 0.41 \cdot \ln y^{+}+5.2$, whereas in the RANS the velocity is underestimated in the viscous sublayer and buffer layer. The heating leads to an upwards shift in the log-law region, which is not represented by the RANS. 
The secondary flow field in the HARCD consists of a counter-rotating vortex pair in each corner, a smaller vortex above the heated wall and a larger one along the lateral wall. Figure $3 b$ depicts the heated wall-normal velocity with the maximum being the footprint of the small vortex and the minimum that of the large vortex in the duct midplane. The small vortex strength is significantly underestimated in the RANS, whereas the large vortex strength agrees well with the LES data. Using other turbulence models than the BSL RSM, the results deviate further from the LES, see [17]. When heating is applied the secondary flow strength becomes weaker along the duct, [19]. The RANS captures this behaviour only for the large vortex, whereas the small vortex strength slightly increases.

In Fig. 3c normalised temperature profiles are shown with $T^{+}=T / T_{\tau}$ and $T_{\tau}=$ $q_{W} /\left(\rho_{W} c_{p_{W}} u_{\tau}\right)$. Kader's law [15] is defined as $T^{+}=\operatorname{Pr} y^{+}$for the viscous sublayer and $T^{+}=2.12 \ln \left(y^{+}\right)+\left(3.85 \operatorname{Pr}^{1 / 3}-1.3\right)^{2}+2.12 \ln (P r)$ for the $\log$-law region, assuming $P r$ and $P r_{t}$ to be constant and pure channel flow. The LES follows Kader's law in the sublayer and shows a significant upwards shift in the log-law region due to the secondary flow presence generating a local hot spot in the midplane. Strong differences between the RANS heat flux closure models become apparent in the loglaw region, however, the temperature is underestimated for all models. One reason is the significantly weaker small vortex. From the BSL RSM with $P r_{t}=0.9$ over $P r_{t}=0.85$ and the algebraic models to the PDE-model, an increasing downwards shift of the $T^{+}$-profile is visible and a reduction of the profile-slope. Overall the $T^{+}$. deviation from the LES grows, accompanied by an increasing deviation of the local and global heat transfer. The integral wall heat flux over the first $500 \mathrm{~mm}$ increases from $3.2 \mathrm{~kW}$ in the LES over $3.6 \mathrm{~kW}$ for BSL RSM with $P r_{t}=0.9$ and $3.9 \mathrm{~kW}$ for the Younis-model to $4.35 \mathrm{~kW}$ for the PDE model. Likewise, the lower wall shear stress is overestimated in the RANS with $\tau_{W, R A N S} \approx 51.0 \mathrm{~Pa}$ for all closure models versus the LES value of $45.7 \mathrm{~Pa}$. Without heating the values show less deviation with $\tau_{W, L E S}=53.2 \mathrm{~Pa}$ and $\tau_{W, R A N S}=54.8 \mathrm{~Pa}$. The observed deviations are possibly due to the usage of the automatic wall treatment option of ANSYS CFX.

The turbulent heat flux comparison in Fig. $3 \mathrm{~d}$ shows, that $\overline{u^{\prime} T^{\prime}}$ is underestimated in the RANS unless the PDE model is employed. The Younis model offers an improvement over the simpler Daly-Harlow model and the $P r_{t}=$ const. models. For the latter $\overline{u^{\prime} T^{\prime}} \approx 0$ due to the negligible streamwise temperature gradient. The $\overline{u^{\prime} T^{\prime}}$ maximum close to the heated wall cannot be represented by the RANS, see [17] for further details. The wall-normal turbulent heat flux is overestimated for all considered RANS models. A similar behaviour as for the temperature and the wall heat flux is observed: the deviation from the LES increases from the $P r_{t}=$ const. models over the algebraic models to the PDE model, providing a further explanation for the overestimated heat transfer in the RANS simulations. 


\section{Channel Flow with Strong Property Variations}

This section focuses on the transcritical channel flow. Results for the RANS BSL RSM in combination with different heat flux closure models are compared to LES to assess the prediction capability of the flow field with strong property variations.

\subsection{Equation System and Numerical Model}

The LES was performed solving the three-dimensional compressible continuity, momentum and total energy equations. The finite-volume method is applied in order to spatially discretise the governing equations on a block structured, curvilinear grid. The compact four cell stencil approach by [10] is used to compute the convective fluxes. A physically consistent subgrid-scale turbulence model based on ALDM [12] is implicitly included in the convective flux calculation. More information about the LES simulation can be found in [9].

The compressible NSE are solved for all transcritical RANS simulations using ANSYS FLUENT [3, 4]. The Reynolds stresses are modelled using the $\omega$-based BSL RSM showing the best results in our preliminary tests. The free stream sensitivity within the BSL RSM model is removed by scaling the baseline $\kappa-\omega$ equations. The $\omega$-equation can be integrated throughout the viscous sublayer allowing for a blending between the viscous sublayer and logarithmic layer formulation. The turbulent heat flux is modelled establishing a relationship between the eddy diffusivity and turbulent Prandtl number. In this study we used $P r_{t}=0.85$ (default in FLUENT) and an algebraic formulation by Kays and Crawford (KC) [21].

Thermodynamic and transport properties are obtained using an adaptive lookup table method, which is based on the REFPROP database [23]. This method has been used for the LES and RANS simulations extracting thermodynamic and transport properties from the tabulated look-up database via trilinear interpolation. The accuracy of the extracted values has been shown in [9].

\subsection{Simulation Setup}

A generic channel flow configuration is used to focus this study on transcritical heat transfer and on the impact of non-linear thermodynamic effects on turbulent flows. Periodic boundary conditions are imposed in stream- and spanwise direction, and isothermal no slip boundary conditions are applied at the top and bottom walls. The channel geometry is $2 \pi h \times 2 h \times \pi h$ in the streamwise, wall-normal and spanwise direction, respectively, see Fig. 4. The channel half-height $h$ is used as characteristic length. A hyperbolic stretching law is applied in wall-normal direction in order to 


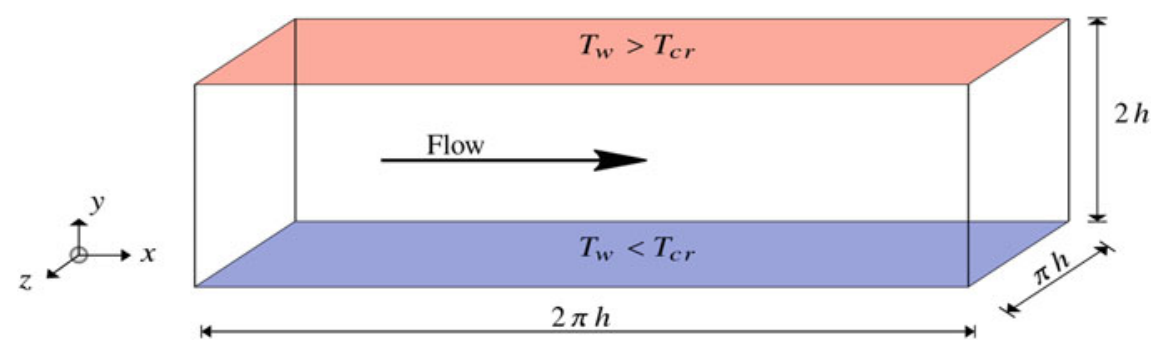

Fig. 4 Computational domain with a hot wall at the top and a cold wall at the bottom at supercritical pressure

fulfill the resolution requirements at walls, whereas a uniform grid spacing is used in the stream- and spanwise direction. Roughness and gravity effects are not considered in the simulations.

Methane is used as working fluid with its critical pressure of $p_{c r}=4.5992 \mathrm{MPa}$ and critical temperature of $T_{c r}=190.564 \mathrm{~K}$. The bulk pressure is $p_{b} \approx 5.0 \mathrm{MPa}$, corresponding to a reduced pressure of $p_{r}=p_{b} / p_{c r}=1.09$. The cold wall temperature is set to $T_{w_{\mathrm{c}}}=180 \mathrm{~K}\left(T_{w_{\mathrm{c}}}<T_{c r}\right)$ and the hot wall temperature to $T_{w_{\mathrm{h}}}=400 \mathrm{~K}$ $\left(T_{w_{\mathrm{h}}}>T_{c r}\right)$, thus a temperature ratio of $T_{w_{\mathrm{h}}} / T_{w_{\mathrm{c}}}=2.22$ is obtained. These boundary conditions encompass the pseudo-boiling temperature of $T_{p b} \approx 193.6 \mathrm{~K}$ at $p_{b}$ and result in a density ratio of $\rho_{w_{\mathrm{c}}} / \rho_{w_{\mathrm{h}}}=12.0$.

A body force in the momentum and energy equation is added to maintain a constant mass flux, which corresponds to a bulk velocity of $u_{b}=74 \mathrm{~m} / \mathrm{s}$. This results in a bulk Reynolds number of $R e_{b}=\left(u_{b} 2 h \rho_{b}\right) / \mu_{b} \approx 1.67 \cdot 10^{4}$. The LES and RANS simulations are initialised with a parabolic velocity profile. A linear temperature distribution with a bulk pressure of $5 \mathrm{MPa}$ is prescribed to accelerate the convergence and reduce high gradients at the beginning of the simulations. Results obtained with the SST model are used as initial guess for the BSL RSM simulations. Convergence is reached when a RMS target value of $1 \cdot 10^{6}$ is surpassed for the momentum, continuity, total energy and RSM transport equation residuals.

\subsection{Flow and Temperature Field}

In the following, the RANS simulations using the BSL RSM turbulence model together with a constant turbulent Prandtl number and the $\mathrm{KC}$ model are compared with the LES. The mean flow properties in the LES are generated by averaging in time and subsequently in streamwise and spanwise direction after reaching a quasistationary state.

Figure 5a shows the van Driest transformed mean velocity distribution at the cold and hot wall side over wall units. Since the turbulent heat flux model has a minor effect on the velocity similar results for the constant turbulent Prandtl number and 

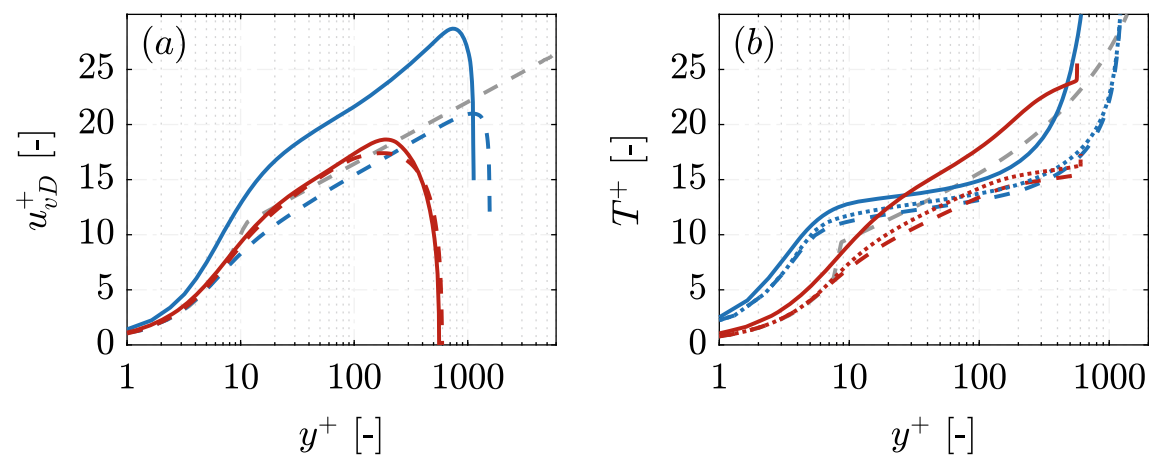

Fig. 5 Mean flow properties over wall units with the van Driest transformed velocity in (a) and transformed temperature in (b) The analytical law of the wall with $\kappa=0.41$ and $B=5.2$, and the empirical function of Kader are plotted as (----). LES (- - ), BSL RSM with $P r_{t}=0.85$ ( ----$)$ and BSL RSM with Kays and Crawford model ( ............. )

the $\mathrm{KC}$ model are achieved. For this reason, the velocity profiles using the $\mathrm{KC}$ model have been excluded in Fig. 5a. A good agreement is observed between the LES and the RANS simulation at the hot wall. The profiles also follow the analytical log law with $\kappa=0.41$ and $B=5.2$, since the fluid exhibits an ideal gas like behavior towards the hot wall. The latter has been shown using the compressibility factor in [9]. The pseudo-boiling position is located at $y^{+} \approx 11$ in the vicinity of the cold wall, where strong property variations are present. As a consequence, the LES and RANS do not coincide and do not follow the law of the wall. Other studies [9, 20, 22, 24] showed, that no general transformation including the semi-local scaling [14] and the transformation by Trettel and Larsson [30] is able to collapse the mean velocity profiles for transcritical flows throughout the viscous sublayer and log law region onto the analytical law of the wall.

The temperature distribution is shown in Fig. $5 \mathrm{~b}$ using $T^{+}$, see Sect.2.3. The distribution at both walls exhibits a viscous sublayer and log law with different slopes. The specific heat capacity peak at the pseudo-boiling position acting as a heat sink leads to a flattening at the cold wall. Only a small difference is observed between RANS and LES, which is slightly improved using the KC model for the turbulent Prandtl number. Due to the strongly varying molecular Prandtl number no analytical law of the wall formulation is included at the cold wall. The RANS and LES profiles at the hot wall diverge with increasing wall distance. The RANS profiles follow the empirical formulation by Kader, see Sect. 2.3, in the sublayer, but underestimate the temperature in the log layer. Using KC model for $\operatorname{Pr}_{t}$ slightly adjusts the temperature towards the Kader law.

The turbulent Prandtl number profiles are compared in Fig. 6. The turbulent Prandtl number in the LES is derived based on the enthalpy since the perfect gas relation $h=c_{p} T$ is not valid in transcritical flows [8]. A good agreement between RANS and LES is achieved at the cold wall for $y^{+}>10$, but the turbulent Prandtl number 

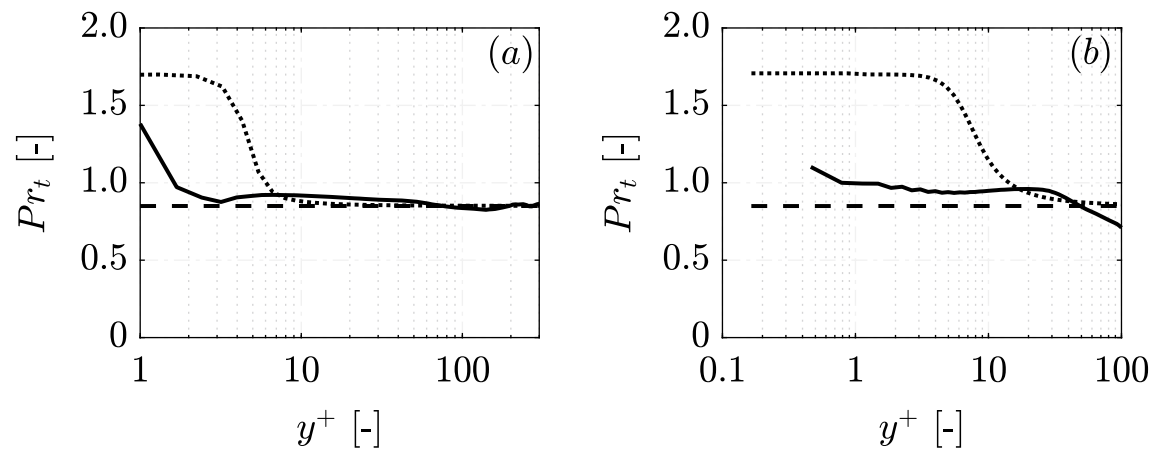

Fig. 6 Turbulent Prandtl number distribution over wall units. $P r_{t}$ at the cold (a) and hot wall (b). LES ( - ), BSL RSM with $P r_{t}=0.85(----)$ and BSL RSM with Kays and Crawford model $(\cdots \cdots \cdots \cdots \cdot \cdots \cdot)$

Table 1 Summary of integral values for LES and RANS simulations

\begin{tabular}{l|l|l|l|l}
\hline & $\tau_{w_{c}}[\mathrm{~Pa}]$ & $\tau_{w_{h}}[\mathrm{~Pa}]$ & $\left|\dot{q}_{w_{c}}\right|\left[\mathrm{MW} \mathrm{m}^{-2}\right]$ & $\left|\dot{q}_{w_{h}}\right|\left[\mathrm{MW} \mathrm{m}^{-2}\right]$ \\
\hline LES & 1257 & 708 & 3.48 & 3.00 \\
\hline $\begin{array}{l}\text { BSL RSM with } \\
P r_{t}=0.85\end{array}$ & 2420 & 802 & 5.26 & 4.95 \\
\hline $\begin{array}{l}\text { BSL RSM with } \\
\text { KC }\end{array}$ & 2410 & 804 & 5.03 & 4.72 \\
\hline
\end{tabular}

in the LES starts to increase closer to wall. This can also be observed at the hot wall, where the $\mathrm{KC}$ formulation increases earlier to the wall value of 1.70.

The evaluation of the integral wall values for the performed simulations in Table 1 shows, that the wall shear stress at the hot wall for the RANS with $\operatorname{Pr}_{t}=0.85$ is close to the LES. The discrepancy in the velocity profiles at the cold wall can also be seen by means of the wall shear stress, which is approximately double as high in the LES. Higher heat flux values in the RANS simulations result in the observed smaller temperature values compared to the LES. The use of $\mathrm{KC}$ for $\operatorname{Pr}_{t}$ does not lead to major improvements in the integral values. Thus, the Reynolds stress modelling has to be analysed and improved as proposed by [20].

\section{Summary and Conclusion}

We have conducted RANS simulations using the BSL RSM in combination with various turbulent heat flux closure models for an asymmetrically heated high aspect ratio water cooling duct and a transcritical channel flow including strong property variations within. For the former we used the commercial solver ANSYS CFX and for the latter ANSYS FLUENT. The results have been compared to well-resolved LES simulations. 
For the HARCD, the BSL RSM was used in combination with $P r_{t}=0.85$, $P r_{t}=0.9$, the algebraic Daly-Harlow and Younis models and additional PDEs for the individual heat flux components. We observed for the secondary flow field, that the small vortex strength and extension is significantly underestimated and that the large vortex strength is in good agreement with the LES. Due to the weaker secondary flow the dimensionless temperature $T^{+}$is underestimated for all RANS model combinations. Using more complex heat flux closure models, the deviation from the LES increases further from the constant $\mathrm{Pr}_{t}$ model over the algebraic models to the PDE model. Likewise, the wall-normal turbulent heat flux $\overline{v^{\prime} T^{\prime}}$ is overestimated and the deviation increases using a more complex heat flux closure. The $T^{+}$-underestimation is accompanied by an overestimation of the local and the global wall heat flux. Similarly the wall shear stresses are overestimated in the RANS with a higher deviation for the heated than the adiabatic duct. A possible reason is the usage of the automatic wall treatment option by ANSYS CFX.

For the transcritical channel case thermodynamic and transport properties have been modelled using the look-up table method. The BSL RSM turbulence model has been used in combination with a constant turbulent Prandtl number of 0.85 and the formulation by Kays and Crawford as heat flux closure. The van Driest transformed velocity profiles show a good agreement between RANS and LES following the law of the wall at the hot wall. A discrepancy has been observed at the cold wall, where the pseudo-boiling is present. This mismatch can also be seen in the wall shear stress values. The temperature is flattened at the cold wall due to the heat capacity peak. The temperature profiles in the RANS simulations are underestimated compared to the LES, which is related to the higher wall heat flux values. An improved heat flux closure given by $\mathrm{KC}$ results in only minor improvements in the temperature profiles. These results lead to the conclusion, that the Reynolds stress modelling has to be addressed in order to overcome the mismatch in the vicinity of the pseudo-boiling to achieve the correct wall shear stresses.

Acknowledgements Financial support has been provided by the German Research Foundation (Deutsche Forschungsgemeinschaft - DFG) within the framework of the Sonderforschungsbereich Transregio 40, SFB-TRR40 (Technological foundations for the design of thermally and mechanically highly loaded components of future space transportation systems). Computational resources have been provided by the Leibniz Supercomputing Centre Munich (LRZ).

\section{References}

1. ANSYS, Inc.: ANSYS CFX-Solver Modeling Guide, Release 14.0 (2011)

2. ANSYS, Inc.: ANSYS CFX-Solver Theory Guide, Release 14.0 (2011)

3. ANSYS, Inc.: ANSYS Fluent, Release 19.2, HelpSystem, Theory Guide

4. ANSYS, Inc.: ANSYS Fluent, Release 19.2, HelpSystem, User's Guide

5. Banuti, D.T., Raju, M., Ma, P.C., Ihme, M., Hickey, J.P.: Seven questions about supercritical fluids-towards a new fluid state diagram. In: 55th AIAA Aerospace Sciences Meeting, 2017$1106(2017)$ 
6. Choi, H.S., Park, T.S.: The influence of streamwise vortices on turbulent heat transfer in rectangular ducts with various aspect ratios. International Journal of Heat and Fluid Flow 40, 1-14 (2013)

7. Daly, B.J., Harlow, F.H.: Transport equations in turbulence. Physics of Fluids 13(11), 26342649 (1970)

8. Doehring, A., Schmidt, S., Adams, N.: Large-eddy simulation of turbulent channel flow at transcritical states. In: Eleventh International Symposium on Turbulence and Shear Flow Phenomena (TSFP11), Southampton (2019)

9. Doehring, A., Schmidt, S., Adams, N.: Numerical Investigation of Transcritical Turbulent Channel Flow. In: 2018 Joint Propulsion Conference, Cincinnati (2018)

10. Egerer, C.P., Schmidt, S.J., Hickel, S., Adams, N.A.: Efficient implicit LES method for the simulation of turbulent cavitating flows. Journal of Computational Physics 316, 453-469 (2016)

11. Gavrilakis, S.: Numerical simulation of low-Reynolds-number turbulent flow through a straight square duct. Journal of Fluid Mechanics 244, 101-129 (1992)

12. Hickel, S., Egerer, C.P., Larsson, J.: Subgrid-scale modeling for implicit large eddy simulation of compressible flows and shock-turbulence interaction. Physics of Fluids 26, (2014)

13. Hickel, S., Adams, N.A., Domaradzki, J.A.: An adaptive local deconvolution method for implicit LES. Journal of Computational Physics 213(1), 413-436 (2006)

14. Huang, P.G., Coleman, G.N., Bradshaw, P.: Compressible turbulent channel flows: DNS results and modelling. Journal of Fluid Mechanics pp. 185-218 (1995)

15. Kader, B.: Temperature and concentration profiles in fully turbulent boundary layers. International Journal of Heat and Mass Transfer 24, 1541-1544 (1981)

16. Kaller, T., Hickel, S., Adams, N.: LES of an Asymmetrically Heated High Aspect Ratio Duct at High Reynolds Number at Different Wall Temperatures. In: 2018 Joint Thermophysics and Heat Transfer Conference, Atlanta (2018)

17. Kaller, T., Hickel, S., Adams, N.: Prediction Capability of RANS Turbulence Models for Asymmetrically Heated High-Aspect-Ratio Duct Flows. In: 2020 AIAA SciTech Forum, Orlando (2020)

18. Kaller, T., Pasquariello, V., Hickel, S., Adams, N.: Large-eddy simulation of the high-Reynoldsnumber flow through a high-aspect-ratio cooling duct. In: Proceedings of the 10th International Symposium on Turbulence and Shear Flow Phenomena (TSFP-10), Chicago (2017)

19. Kaller, T., Pasquariello, V., Hickel, S., Adams, N.A.: Turbulent flow through a high aspect ratio cooling duct with asymmetric wall heating. Journal of Fluid Mechanics 860, 258-299 (2019)

20. Kawai, S.: Heated transcritical and unheated non-transcritical turbulent boundary layers at supercritical pressures. Journal of Fluid Mechanics 865, 563-601 (2019)

21. Kays, W.M., Crawford, M.E.: Convective Heat and Mass Transfer, 3rd edn. McGraw-Hill, Inc. (1993)

22. Kim, K., Hickey, J.P., Scalo, C.: Pseudophase change effects in turbulent channel flow under transcritical temperature conditions. Journal of Fluid Mechanics 871, 52-91 (2019)

23. Lemmon, E.W., Huber, M.L., McLinden, M.O.: NIST Standard Reference Database 23: Reference Fluid Thermodynamic and Transport Properties-REFPROP, Version 9.1. National Institute of Standards and Technology (2013)

24. Ma, P.C., Yang, X.I.A., Ihme, M.: Structure of wall-bounded flows at transcritical conditions. Physical Review Fluids 3(3), 1-24 (2018)

25. Pirozzoli, S., Modesti, D., Orlandi, P., Grasso, F.: Turbulence and secondary motions in square duct flow. Journal of Fluid Mechanics 840, 631-655 (2018)

26. Pizzarelli, M., Nasuti, F., Onofri, M.: Numerical Analysis of Three-Dimensional Flow of Supercritical Fluid in Asymmetrically Heated Channels. AIAA Journal 47(11), 2534-2543 (2009)

27. Pizzarelli, M., Nasuti, F., Onofri, M.: Trade-off analysis of high-aspect-ratio-cooling-channels for rocket engines. International Journal of Heat and Fluid Flow 44, 458-467 (2013)

28. Rochlitz, H., Scholz, P., Fuchs, T.: The flow field in a high aspect ratio cooling duct with and without one heated wall. Experiments in Fluids 56(12), 1-13 (2015)

29. Salinas-Vásquez, M., Métais, O.: Large-eddy simulation of the turbulent flow through a heated square duct. Journal of Fluid Mechanics 453, 201-238 (2002) 
30. Trettel, A., Larsson, J.: Mean velocity scaling for compressible wall turbulence with heat transfer. Physics of Fluids 28(2), 026,102 (2016)

31. Vinuesa, R., Noorani, A., Lozano-Duran, A., El Khoury, G., Schlatter, P., Fischer, P.F., Nagib, N.M.: Aspect ratio effects in turbulent duct flows studied through direct numerical simulation. Journal of Turbulence 15(10), 677-706 (2014)

32. Younis, B.A., Speziale, C.G., Clark, T.T.: A rational model for the turbulent scalar fluxes. Proceedings of the Royal Society A: Mathematical, Physical and Engineering Sciences 461(2054), 575-594 (2005)

Open Access This chapter is licensed under the terms of the Creative Commons Attribution 4.0 International License (http://creativecommons.org/licenses/by/4.0/), which permits use, sharing, adaptation, distribution and reproduction in any medium or format, as long as you give appropriate credit to the original author(s) and the source, provide a link to the Creative Commons license and indicate if changes were made.

The images or other third party material in this chapter are included in the chapter's Creative Commons license, unless indicated otherwise in a credit line to the material. If material is not included in the chapter's Creative Commons license and your intended use is not permitted by statutory regulation or exceeds the permitted use, you will need to obtain permission directly from the copyright holder. 